\title{
Primary extragastrointestinal stromal tumor of the pleura: A case report
}

\author{
CAI-QING ZHANG ${ }^{1}$, DE-GAN LU ${ }^{1}$, QING-FA LIU ${ }^{1}$ and WEI XIAO ${ }^{2}$ \\ ${ }^{1}$ Department of Respiratory Medicine, Shandong Provincial Qianfoshan Hospital Affiliated to Shandong University, Jinan, \\ Shandong 250014; ${ }^{2}$ Department of Respiratory Medicine, Qilu Hospital of Shandong University, \\ Jinan, Shandong 250012, P.R. China
}

Received January 24, 2015; Accepted February 25, 2016

DOI: $10.3892 / 01.2016 .4344$

\begin{abstract}
Gastrointestinal stromal tumors (GISTs) are the most common type of mesenchymal tumor of the gastrointestinal tract. The stomach and small intestine are the most common sites of occurrence. GISTs are mesenchymal neoplasms originating from the interstitial cells of Cajal (ICCs), and are characterized by positivity for cluster of differentiation (CD) 117, also known as proto-oncogene c-Kit. While the majority of GISTs develop in the alimentary tract, in rare cases they may also be found in extragastrointestinal tissues. This type of GIST is known as an extragastrointestinal stromal tumor (EGIST). Despite the fact that EGISTs have been reported in the mesentery, omentum and retroperitoneum, primary intrathoracic EGISTs, arising from the pleura or lungs, are rare. The patient presented in the current study was a 40-year-old man, who presented with a cough and pyrexia, with pleural effusion on the left side. Multiple nodules throughout the parietal pleura were identified by thoracoscopy and a diagnosis of primary GIST of pleura was established, since they were positive for CD117 and discovered on GIST-1 and there was no evidence of gastrointestinal tumors. Subsequently, the patient was administered with imatinib and had no signs of disease recurrence 2 years later.
\end{abstract}

\section{Introduction}

Gastrointestinal stromal tumors (GISTs) comprise the most common type of mesenchymal neoplasm arising from the

Correspondence to: Dr De-Gan Lu, Department of Respiratory Medicine, Shandong Provincial Qianfoshan Hospital Affiliated to Shandong University, 16766 Jingshi Road, Jinan, Shandong 250014, P.R. China

E-mail: deganlu@126.com

Professor Wei Xiao, Department of Respiratory Medicine, Qilu Hospital of Shandong University, 107 Wenhua West Road, Jinan, Shandong 250012, P.R. China

E-mail: xiaowei@163.com

Key words: extragastrointestinal stromal tumors, pleura, biopsy, thoracoscopy interstitial cells of Cajal (ICCs) of the myenteric plexus. GISTs account for $0.1-3 \%$ of all gastrointestinal malignancies (1). The ICCs in the muscularis propria and myenteric plexus are known as the pacemaker cells of the gastrointestinal tract (2). GISTs have been demonstrated to express the receptor tyrosine kinase protein cluster of differentiation (CD)117, also known as proto-oncogene c-Kit (3). GISTs primarily arise from the stomach (60-70\%) and between the ileum and jejunum (25-30\%), and are observed less frequently in the colorectum (5-15\%), duodenum (5\%) and esophagus (2\%) (4).

GISTs may additionally be observed in the omentum, mesentery, retroperitoneum, pancreas and pelvis, as well as adjacent to, but separate from, the stomach and intestine (5-7). GISTs identified in the aforementioned locations have been termed extragastrointestinal stromal tumors (EGISTs), as they occur outside the gastrointestinal tract. The clinicopathological and molecular profiles of EGISTs are similar to those of GISTs (8). EGISTs have additionally been reported in the gallbladder, prostate and rectovaginal septum (9). To the best of our knowledge, only a small number of cases of primary EGISTs originating from pleuropulmonary sites have been previously reported $(10,11)$.

The present study describes a rare case of primary EGIST originating from the right pleural cavity of a 40-year-old man. The diagnosis was confirmed by thoracoscopy and immunohistochemistry.

\section{Case report}

A 40-year-old man was admitted on October 17, 2013 to the Department of Respiratory Medicine, Qianfoshan Hospital Affiliated to Shandong University (Jinan, China) presenting with a moderate cough, which was progessive, and pyrexia that had been ongoing for one month. The patient had been expectorating $\sim 10 \mathrm{ml}$ of grossly frothy sputum per day, without hemoptysis or emaciation. Temperature, pulse, respiratory rate and blood pressure were $38.2^{\circ} \mathrm{C}$ (normal range, $37^{\circ} \mathrm{C}$ ), 100 beats/min (normal range, $60-100$ beats $/ \mathrm{min}$ ), 20 times $/ \mathrm{min}$ (normal range, 16-18 beats/min) and $128 / 85 \mathrm{mmHg}$ (normal range, $90-140 / 60-90 \mathrm{mmHg}$ ), respectively. Laboratory tests performed upon admission revealed the following: White blood cell count, 14.9x10 $/ 1$ (normal range, $4-10 \times 10^{9} / 1$ ); segmented neutrophils, $78 \%$ (normal range, 50-70\%); hemoglobin 


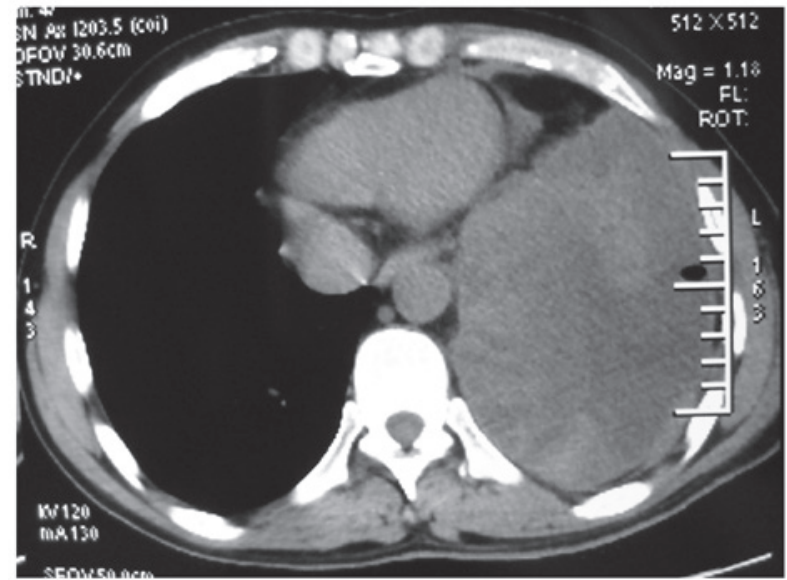

Figure 1. Computed tomography scan of the chest revealing a left-sided pleural effusion.

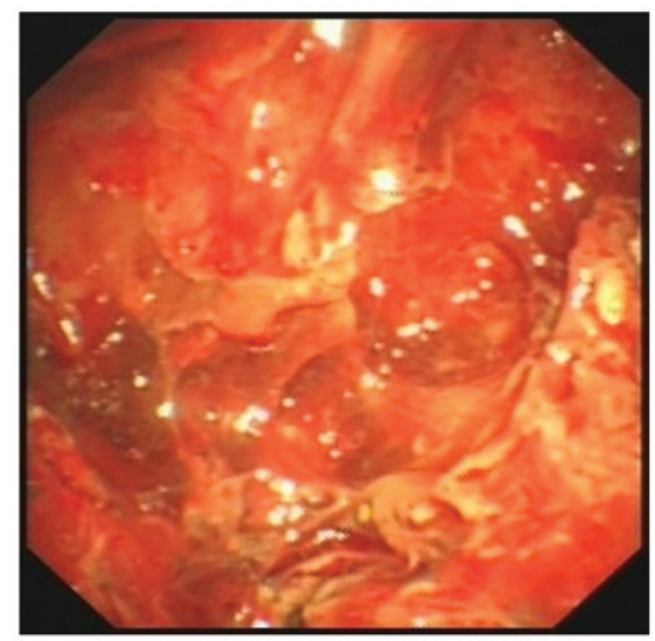

Figure 2. Thoracoscopic image revealing multiple nodules of varying size in the parietal pleura.

concentration, $132 \mathrm{~g} / 1$ (normal range, 120-170 g/l); erythrocyte sedimentation rate, $87 \mathrm{~mm}$ in the first hour (normal range, $0-15 \mathrm{~mm} / \mathrm{h}$ ); C-reactive protein concentration, $173 \mathrm{mg} / \mathrm{l}$ (normal range, 0-10 mg/l). A computed tomography (CT) (Discovery CT 750 HD; GE Healthcare, Milwaukee, WI, USA) scan of the chest revealed left-sided pleural effusion (Fig. 1). Bacterial (including mycobacterial) and fungal examinations of the repeated sputum were negative. The purified protein derivative skin test was also negative.

A closed drainage of the pleural cavity was performed on day 2 following admission. Approximately $500 \mathrm{ml}$ pale red and turbid fluid was drained from the left pleural cavity. The fluid was negative for bacterial, fungal or acid-fast organisms, and no malignant cells were identified. Biochemical analysis of the pleural fluid revealed the following: Adenosine deaminase, $38.2 \mathrm{U} / 1$ (normal range, $0-25 \mathrm{U} / 1$ ); total protein, $41.3 \mathrm{~g} / 1$ (normal range, $0-30 \mathrm{~g} / \mathrm{l}$ ); glucose, $2.58 \mathrm{mmol} / 1$ (normal range, 3.9-6.1 mmol/1); lactate dehydrogenase 3,422.8 U/1 (normal range, 109-245 U/1). Thoracoscopy (LTF 240; Olympus Corporation, Tokyo, Japan) identified multiple nodules of varying size throughout the parietal pleura of the patient (Fig. 2). A

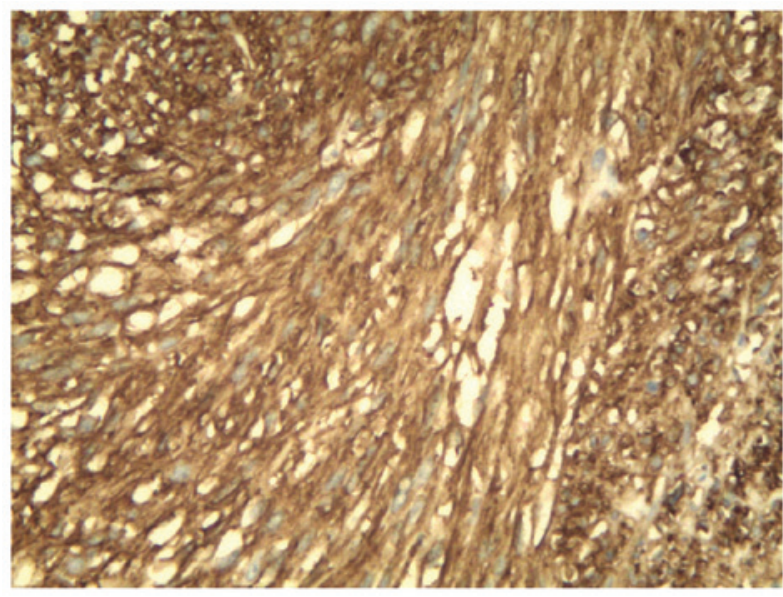

Figure 3. Immunohistochemical staining of tissue from a transthoracoscopic biopsy demonstrated positivity for cluster of differentiation 117 (magnification, $\mathrm{x} 200$ ).

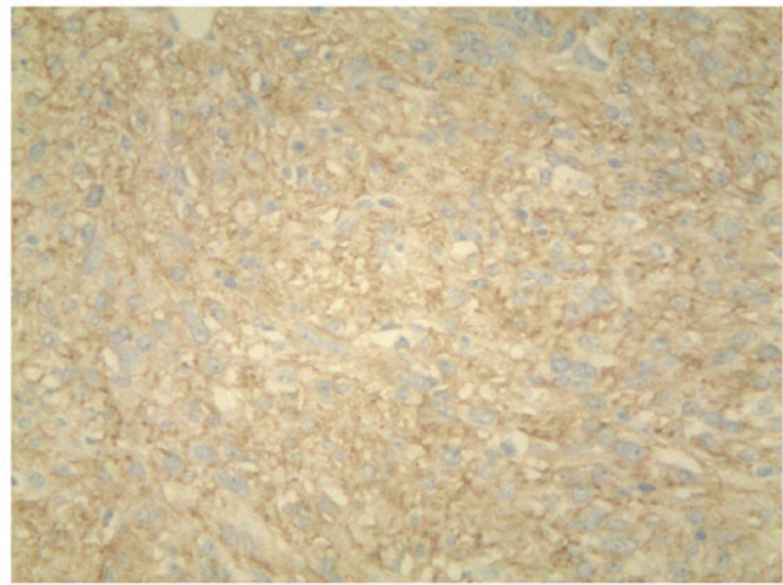

Figure 4. Immunohistochemical staining of tissue from a transthoracoscopic biopsy demonstrated positivity for discovered on GIST-1 (magnification, $\mathrm{x} 200$ ).

transthoracoscopic biopsy of the nodules was performed, and formalin-fixed and paraffin-embedded tissues were sliced into $4-\mu \mathrm{m}$ sections on glass slides.

Immunohistochemitry was performed as follows: The tissue sections were deparaffinized in xylene and rehydrated in graded alcohol dilutions. Endogenous peroxidase activity was blocked with $3 \% \mathrm{H}_{2} \mathrm{O}_{2}$ at room temperature for $10 \mathrm{~min}$. Subsequently, the tissues were fixed by heating at $95^{\circ} \mathrm{C}$ for 30 min and incubated overnight at $4^{\circ} \mathrm{C}$ with primary rabbit monoclonal antibodies from Cell Marque ${ }^{\mathrm{TM}}$ (Sigma-Aldrich, St. Louis, MO, USA) at a dilution of 1:200 against CD117 (catalog no. YR145) and discovered on GIST-1 (DOG-1; catalog no. SP31). The tissues were incubated for $20 \mathrm{~min}$ at room temperature with a secondary antibody (catalog no. f03931; Roche Diagnostics, Shanghai, China). Following staining for 3 min with 3,3'-Diaminobenzidine Substrate (Roche Diagnostics), the tissues were counterstained with hematoxylin (Roche Diagnostics). Negative controls were performed by replacing the primary antibody with phosphate-buffered saline. Immunohistochemistry revealed long spindle cells arranged in a palisade or bundle arrangement, and positive staining for 
CD117 (Fig. 3) and DOG1 (Fig. 4) was observed. No tumors were identified by fibergastroscopy or fibercoloscopy in the esophagus, stomach and colorectum. The abdominal CT scan excluded the possibility for pleural GIST metastases. The patient received treatment of $400 \mathrm{mg}$ imatinib mesylate daily for one year, following a presumptive diagnosis of EGIST. The patient was examined with chest CT every 3 months and no evidence of disease recurrence was found. At the time of writing the present study, the patient remained alive with no signs of disease recurrence.

Written informed consent was obtained from the patient for the publication of the present study.

\section{Discussion}

The present study reports a case of a 40-year-old man with multiple primary EGISTs in the left pleural cavity. The diagnosis was verified by immunohistochemical staining for CD117 and DOG1, which are known to be markers for GISTs (12).

GISTs, the most commonly observed mesenchymal neoplasms of the gastrointestinal tract, originate from ICCs or associated CD117-positive-stem cell-like precursors $(13,14)$. The molecular pathogenesis of GISTs is typically driven by mutations of the Kit gene that encodes CD117 (15). In addition, CD34 has been demonstrated to exhibit strong and diffuse staining in $\sim 70 \%$ of GIST cases (16). GISTs may also be positive for smooth muscle actin, and negative for desmin and S-100 protein (7). GISTs are able to occur in ICCs located anywhere along the gastrointestinal tract, including the esophagus, stomach, duodenum, small intestine and colorectum (17).

Primary EGISTs are rare, and typically manifest as enlarging masses of variable duration in adults (18). In $80 \%$ of cases, EGISTs are observed in the omentum and mesentery (19). There have additionally been reports of EGISTs in the pleura, pancreas and abdominal wall (20). The origin of EGISTs remains to be elucidated; however, GISTs and EGISTs have been confirmed to share various clinicopathological and histological features (11). As the presence of GISTs have been observed in numerous organs, including the urinary bladder (21), prostate (22), uterus (23), gall bladder (24) and myocardium (25), it can be assumed that EGISTs originate from common precursor cells that are able to differentiate into ICC-derived neoplasms outside of the gastrointestinal tract during development (26). An alternative explanation is that this type of tumor originates from the pluripotent mesenchymal stem cells located outside the gastrointestinal tract (10).

The clinical implications of EGISTs remain to be fully elucidated. CT scanning is commonly used for the detection, staging, surgical planning and follow-up of patients with EGISTs (10). EGISTs should be considered in the differential diagnosis of mesenchymal tumors, and immunohistochemistry should be used to confirm the diagnosis (27). CD117 is the most specific diagnostic marker for EGISTs, and may be used to detect the product of the proto-oncogene c-kit by immunohistochemical staining (28). The majority of GISTs are positive for CD117; only 5\% demonstrate negativity (29). Immunohistochemical analysis of these markers is being used as an alternative to expensive mutation analysis. The positivity of an additional marker, DOG1, has been shown to have a significant role in determining the diagnosis and prognosis in EGISTs (30).

The prognosis of patients with EGISTs is believed to be poorer than that of patients with GISTs (5), due to the fact that EGISTs are frequently accompanied by adverse prognostic factors, including high proliferative indices, large and distant metastases, and lymph node involvement (11). Approximately $50 \%$ of patients with EGISTs have been reported to develop metastasis or succumb to the primary tumors; therefore, EGISTs should be considered as highly aggressive and be treated as high-grade intra-abdominal sarcomas (6). The primary treatment for EGISTs is surgical resection. Imatinib mesylate has been observed to be beneficial for induction and adjuvant therapy (10). An increased number of therapeutic strategies, that include the incorporation of molecular features, are required to be established for the management of this disease.

In summary, the current study presented a rare case of GIST arising from the pleural cavity. The diagnosis was based on pathological results and immunohistochemical findings, which included expression of CD117 and DOG-1. EGISTs should be acknowledged in the differential diagnosis in patients with pleural effusion. Additional data regarding disease characterization, treatment and prognosis are required for this rare disease.

\section{Acknowledgements}

The authors would like to thank Professor Xiaowei (Department of Respiratory Medicine, Qilu Hospital, Shandong University, Jinan, China) for assistance in the preparation of this manuscript.

\section{References}

1. Liegl-Atzwanger B, Fletcher JA and Fletcher CD: Gastrointestinal stromal tumors. Virchows Arch 456: 111-127, 2010.

2. Youm JB, Leem CH, Lee SR, Song IS, Kim HK, Heo HJ, Kim BJ, Kim N and Han J: Modeling of stochastic behavior of pacemaker potential in interstitial cells of Cajal. Prog Biophys Mol Biol 116: 56-69, 2014.

3. Demetri GD, von Mehren M, Blanke CD, Van den Abbeele AD, Eisenberg B, Roberts PJ, Heinrich MC, Tuveson DA, Singer S, Janicek M, et al: Efficacy and safety of imatinib mesylate in advanced gastrointestinal stromal tumors. N Engl J Med 347: 472-480, 2002

4. Emory TS, Sobin LH, Lukes L, Lee DH and O'Leary TJ: Prognosis of gastrointestinal smooth-muscle (stromal) tumors: Dependence on anatomic site. Am J Surg Pathol 23: 82-87, 1999.

5. Miettinen $M$ and Lasota J: Gastrointestinal stromal tumors: Pathology and prognosis at different sites. Semin Diagn Pathol 23: 70-83, 2006.

6. Reith JD, Goldblum JR, Lyles RH and Weiss SW: Extragastrointestinal (soft tissue) stromal tumors: An analysis of 48 cases with emphasis on histologic predictors of outcome. Mod Pathol 13: 577-585, 2000.

7. Miettinen M, Monihan JM, Sarlomo-Rikala M, Kovatich AJ, Carr NJ, Emory TS and Sobin LH: Gastrointestinal stromal tumors/smooth muscle tumors (GISTs) primary in the omentum and mesentery: Clinicopathologic and immunohistochemical study of 26 cases. Am J Surg Pathol 23: 1109-1118, 1999.

8. Yamamoto H, Oda Y, Kawaguchi K, Nakamura N, Takahira T, Tamiya S, Saito T, Oshiro Y, Ohta M, Yao T and Tsuneyoshi M: c-kit and PDGFRA mutations in extragastrointestinal stromal tumor (gastrointestinal stromal tumor of the soft tissue). Am J Surg Pathol 28: 479-488, 2004. 
9. Hirano H, Yoshida T, Yoshimura H, Fukuoka M, Ohmura N, Nishizawa Y, Tachibana S, Hirota S, Zozumi M and Nishigami T: Extra-gastrointestinal stromal tumor of the pelvic cavity: Case report. Med Mol Morphol 45: 173-177, 2012.

10. Long KB, Butrynski JE, Blank SD, Ebrahim KS, Dressel DM, Heinrich MC, Corless CL and Hornick JL: Primary extragastrointestinal stromal tumor of the pleura: Report of a unique case with genetic confirmation. Am J Surg Pathol 34: 907-912, 2010.

11. Yi JH, Sim J, Park BB, Lee YY, Jung WS, Jang HJ, Ha TK and Paik SS: The primary extra-gastrointestinal stromal tumor of pleura: A case report and a literature review. Jpn J Clin Oncol 43: 1269-1272, 2013.

12. Ríos-Moreno MJ, Jaramillo S, Pereira Gallardo S, Vallejo A, Mora M, García-Escudero A, Amérigo J and González-Cámpora R: Gastrointestinal stromal tumors (GISTs): CD117, DOG-1 and $\mathrm{PKC} \theta$ expression. Is there any advantage in using several markers? Pathol Res Pract 208: 74-81, 2012.

13. Kindblom LG, Remotti HE, Aldenborg F and Meis-Kindblom JM Gastrointestinal pacemaker cell tumor (GIPACT): Gastrointestinal stromal tumors show phenotypic characteristics of the interstitial cells of Cajal. Am J Pathol 152: 1259-1269, 1998.

14. Hirota S, Isozaki K, Moriyama Y, Hashimoto K, Nishida T, Ishiguro S, Kawano K, Hanada M, Kurata A, Takeda M, et al: Gain-of-function mutations of c-kit in human gastrointestinal stromal tumors. Science 279: 577-580, 1998.

15. Patil DT and Rubin BP: Genetics of gastrointestinal stromal tumors: A heterogeneous family of tumors? Surg Pathol Clin. 8: 515-524, 2015.

16. Rabin I, Chikman B, Lavy R, Sandbank J, Maklakovsky M, Gold-Deutch R, Halpren Z, Wassermann I and Halevy A: Gastrointestinal stromal tumors: A 19 year experience. Isr Med Assoc J 11: 98-102, 2009.

17. Miettinen $\mathrm{M}$ and Lasota J: Gastrointestinal stromal tumors: Review on morphology, molecular pathology, prognosis, and differential diagnosis. Arch Pathol Lab Med 130: 1466-1478, 2006

18. Iqbal N, Sharma A and Iqbal N: Clinicopathological and treatment analysis of 13 extragastrointestinal stromal tumors of mesentery and retroperitoneum. Ann Gastroenterol 28: 105-108, 2015.

19. Gowrishankar S: Epithelioid omental extra-gastrointestinal stromal tumor: Report of a case. Indian J Pathol Microbiol 54: 618-619, 2011.

20. Alkhatib L, Albtoush O, Bataineh N, Gharaibeh K, Matalka I and Tokuda Y: Extragastrointestinal Stromal Tumor (EGIST) in the abdominal wall: Case report and literature review. Int J Surg Case Rep 2: 253-255, 2011.
21. Mekni A, Chelly I, Azzouz H, Ben Ghorbel I, Bellil S, Haouet S, Kchir N, Zitouna M and Bellil K: Extragastrointestinal stromal tumor of the urinary wall bladder: Case report and review of the literature. Pathologica 100: 173-175, 2008.

22. Anagnostou E,Miliaras D and Panagiotakopoulos V: Diagnosis of gastrointestinal stromal tumor (GIST) on transurethral resection of the prostate: A case report and review of the literature. Int J Surg Pathol 19: 632-636, 2011.

23. Terada T: Gastrointestinal stromal tumor of the uterus: A case report with genetic analyses of c-kit and PDGFRA genes. Int J Gynecol Pathol 28: 29-34, 2009.

24. Bolanaki H, Delladetsima I, Argyropoulou P, Kapranou A, Kakolyris S, Simopoulos C and Karayiannakis AJ: Primary Malignant Gastrointestinal Stromal Tumor (GIST) of the Gallbladder: Report of a Case. J Gastrointest Cancer 43 (Suppl 1): 151-155. 2012.

25. Bashir U, Qureshi A, Khan HA and Uddin N: Gastrointestinal stromal tumor with skeletal muscle, adrenal and cardiac metastases: An unusual occurrence. Indian J Pathol Microbiol 54: 362-364, 2011.

26. Casella C, Villanacci V, D'Adda F, Codazzi M and Salerni B: Primary extra-gastrointestinal stromal tumor of retroperitoneum. Clin Med Insights Oncol 6: 189-197, 2012.

27. Patnayak R, Jena A, Parthasarathy S, Prasad PD, Reddy MK, Chowhan AK, Rukamangadha N and Phaneendra BV: Primary extragastrointestinal stromal tumors: A clinicopathological and immunohistochemical study - a tertiarycare center experience. Indian J Cancer 50: 41-45, 2013.

28. Joensuu H, Roberts PJ, Sarlomo-Rikala M, Andersson LC, Tervahartiala P, Tuveson D, Silberman S, Capdeville R, Dimitrijevic S, Druker B and Demetri GD: Effect of the tyrosine kinase inhibitor STI571 in a patient with a metastatic gastrointestinal stromal tumor. N Engl J Med 344: 1052-1056, 2001.

29. Xu C, Han H, Wang J, Zhang B, Shao Y, Zhang L, Wang H, Wang H, Wu Y, Li X, et al: Diagnosis value of CD117 and PDGFRA, alone or in combination DOG1, as biomarkers for gastrointestinal stromal tumors. Ann Transl Med 3: 308, 2015.

30. Kim KH, Nelson SD, Kim DH, Choi KU, Kim SJ, Min KW, Jang KS, Paik SS, Oh YH, Chae SW, et al: Diagnostic relevance of overexpressions of PKC- $\theta$ and DOG- 1 and KIT/PDGFRA gene mutations in extra-gastrointestinal stromal tumors: A Korean six-centers study of 28 cases. Anticancer Res 32: 923-937, 2012. 